\title{
APPLICATION OF COMBINATION OF NEURAL NETWORKS AND FUZZY LOGIC IN PLANNING OF MOBILE ROBOT MOTION IN UNKNOWN ENVIRONMENT
}

\author{
Andrius Nemeiksis, Vitalijs Osadcuks \\ Latvia University of Life Sciences and Technologies, Latvia \\ nemeiksis.andrius@1lu.lv, vtl@tvnet.lv
}

\begin{abstract}
The significance of automated control systems to contemporary society is profound, as large-scale industrial robots are used in industrial plants, autonomous vacuum cleaners are applied at home, and autonomous cars are driven in the streets. Regardless of the nature of the robot, one of its main tasks is to do no harm to the person and the environment, and that is the greatest problem of robotics - orientation. Therefore, the problem of orientation of autonomous robots is being solved by a robot exploring the environment, remembering the space and time-varying environmental changes. On that ground, the task of this article is to develop such scientific researches, which define the mobile robot motion in an unknown environment, based on fuzzy logic and the analysis of neural networks, setting an aim to work out methods for developing intellectual systems for planning a mobile robot motion fuzzy logic and neural networks to ensure that the robot performs the planned and adjusted on the way safe trajectory in an environment with unknown obstacles. Therefore, the entire study in the article is aimed at the analysis of fuzzy logic that is analysed as the entirety of the mathematical description methods of fuzzy sets with the formalization of logical conclusions from the fuzzy assumptions, as in this case the decision-making mechanism always allows the generation of the robot's responsive motions caused by the appearance of obstacles in its trajectory; as well as the analysis of neural networks, which links between neurons determine the complexity and flexibility of the operation of the entire neural network, by addressing the problem of planning a mobile robot motion in an unknown environment and which basically depends upon the level of specific network training. As a result of the study, the method of a mobile robot motion in an unknown dynamic environment was created by using a multi-agent system, involving a combination of neural networks and fuzzy blocks.
\end{abstract}

Keywords: neural network, unknown environment, multi-agent system, fuzzy logic, static and dynamic obstacles, planned track.

\section{Introduction}

Thanks to the breakthroughs in computing and technologies, the progress of robotics has been given more and more importance in the specialised spheres of social activities as well as in the performance of various operations [1-3]. In addition, the potential of robotics in improving the quality of people's daily life and making it more comfortable is underlined. Consequently, the development of an effective robot control system, which is currently the most urgent and topical question of this sphere, is being increasingly raised all over the world: international seminars and conferences are held to analyse not only the latest achievements in robot control systems but also their development prospects [4-6].

The significance of the experimental section of this thesis lays in the necessity to confirm the efficient functioning of the principal system of mobile robot movement in an unknown environment developed in practice, which prevents from colliding with the unknown dynamic obstacles in the working area of the robot moving according to the planned trajectory. For the above-mentioned reason, when conducting the experiment, the mobile robot must move from the start point to the destination point without having information about the environment. Therefore, planning of the mobile robot movement along the trajectory safe from collisions with unknown obstacles is based on the principle of the system's response to the obstacles occurring on the planned route.

\section{Materials and methods}

The functional scheme of the experimental system is presented in Fig. 1. Based on this scheme, the sensors initially provide information about the distance between the robot and the obstacles located in its operation area; also, the signals for the speed of each actuator are read. Later, this information gets into electronic input and output interface modules (Arduino Mega sensor shield v2.0 [7]). This process uses control signals generated by PC and transmitted through the serial port to the electronic input and output interface modules. Later, digitized distance values get into the micro-controller through this port, and from there to the computer via a USB cable or wireless module [8;9]. It should be noted that these values, changed by a specific figure, are the input parameters of the control 
algorithm. And the mobile robot position change commands are directly transmitted to the drive through the input and output interface module to the control motors [10], enabling the robot to move along a safe trajectory are the outputs of the control algorithm. Fig. 1 presents the mobile robot used for the experimental research with the installed distance and position sensors.

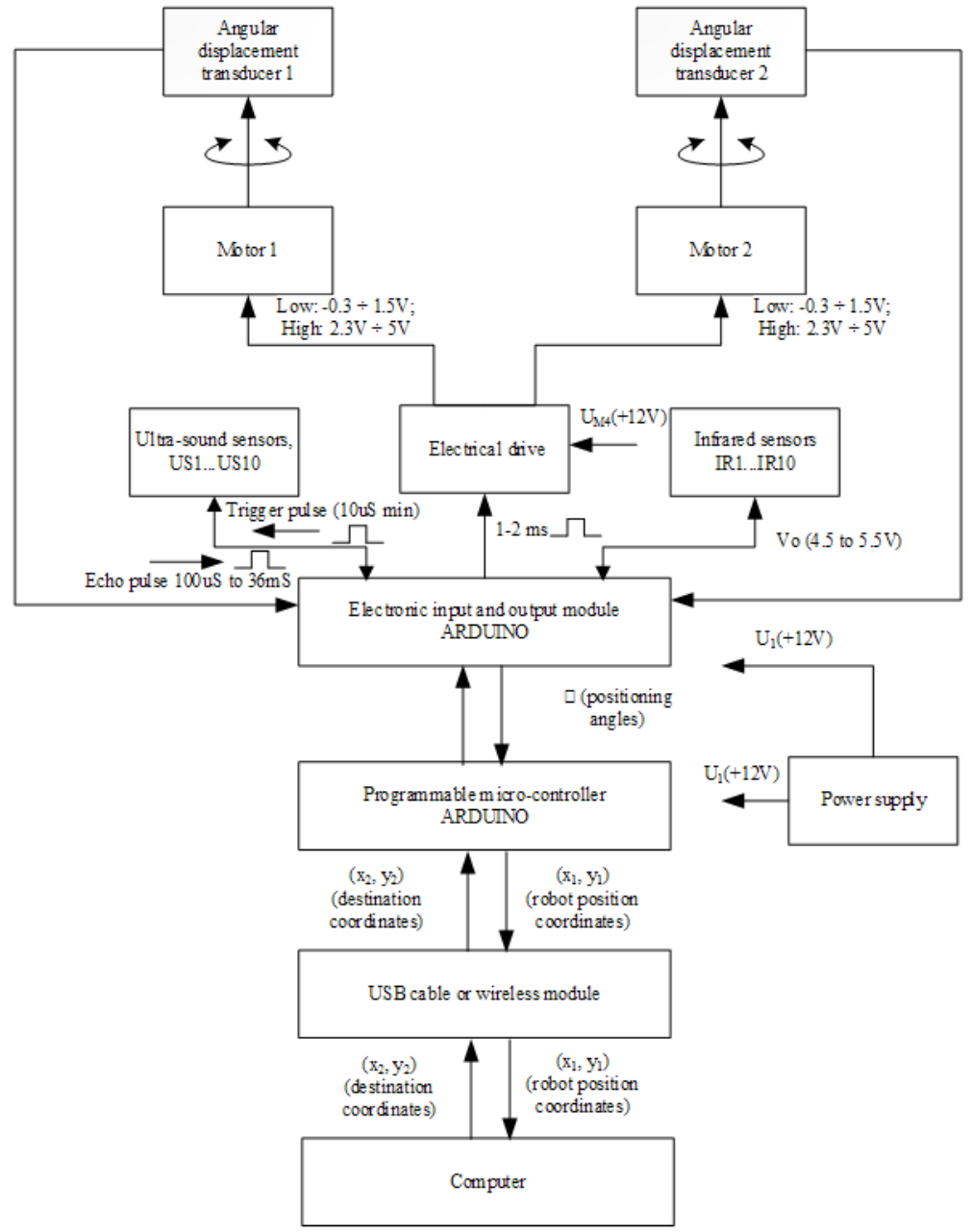

Fig. 1. Functional scheme of experimental system

Using the operational control algorithm, the mobile robot movement is directly based on the realtime control commands that are provided during each iteration of the program, and which are the reaction to the changes in the unknown environment. Motor control commands used in the operational control algorithm are formed with respect to the changes in the parameters ddestination ( $\Delta \mathrm{x}$ and $\Delta \mathrm{y})$ and $\mu$.

These parameters can be provided by the intelligent system for planning the mobile robot movement in an unknown dynamic environment using a multi-agent system based on processing the information concerning the robot and the surrounding unknown dynamic environment with a help of a three-stage process. The structure of this system is presented in Fig. 2.

During the first stage of planning, the distance $(\mathrm{d} S 1)$ between the robot and the obstacles located in its operating area as well as the safe distance (dsafe) are established; also, the possible position of obstacles is classified based on the information from ultra-sound and infra-red distance sensors with their model being in a form of a multi-layer neural network trained using the method of error back propagation in autonomous mode.

During the second stage, obstacle-bypass, wall-tracking, movement-to-destination and speed control agents are developed using fuzzy blocks. Signals of the sensors US2-IR2, US3-IR3, US4-IR4 or US7-IR7, US8-IR8, US9-IR9 are the outputs of the first fuzzy block (1FB), which performs the role of the obstacle-bypass agent. The second fuzzy block (2FB) - wall tracking agent - receives signals from the sensors US1-IR1, US10-IR10 or US5-IR5, US6-IT6. Movement-to-destination agent is based 
on the third fuzzy block (3FB), when there are no obstacles around the robot in the sensor operation area. Fuzzy block outputs are the mobile robot rotation angles $\mu 1, \mu 2$ and $\mu 3$.

During the third stage, the speed control agent is developed using the fourth fuzzy block (4FB). Based on the results obtained during the first and second stages, the final value of the mobile robot rotation angle change $-\mu$ is determined corresponding to each of the agents. Mobile robot rotation angle and the distance between the robot and the destination point are the inputs of the fourth fuzzy block (4FB). Outputs of the 4FB: signal controlled by the drives, which help avoid collisions of the robot with unknown obstacles and reach the destination point.

Fig. 2 presents the operational control algorithm for the modified intelligent system for planning the mobile robot movement in unknown environment.

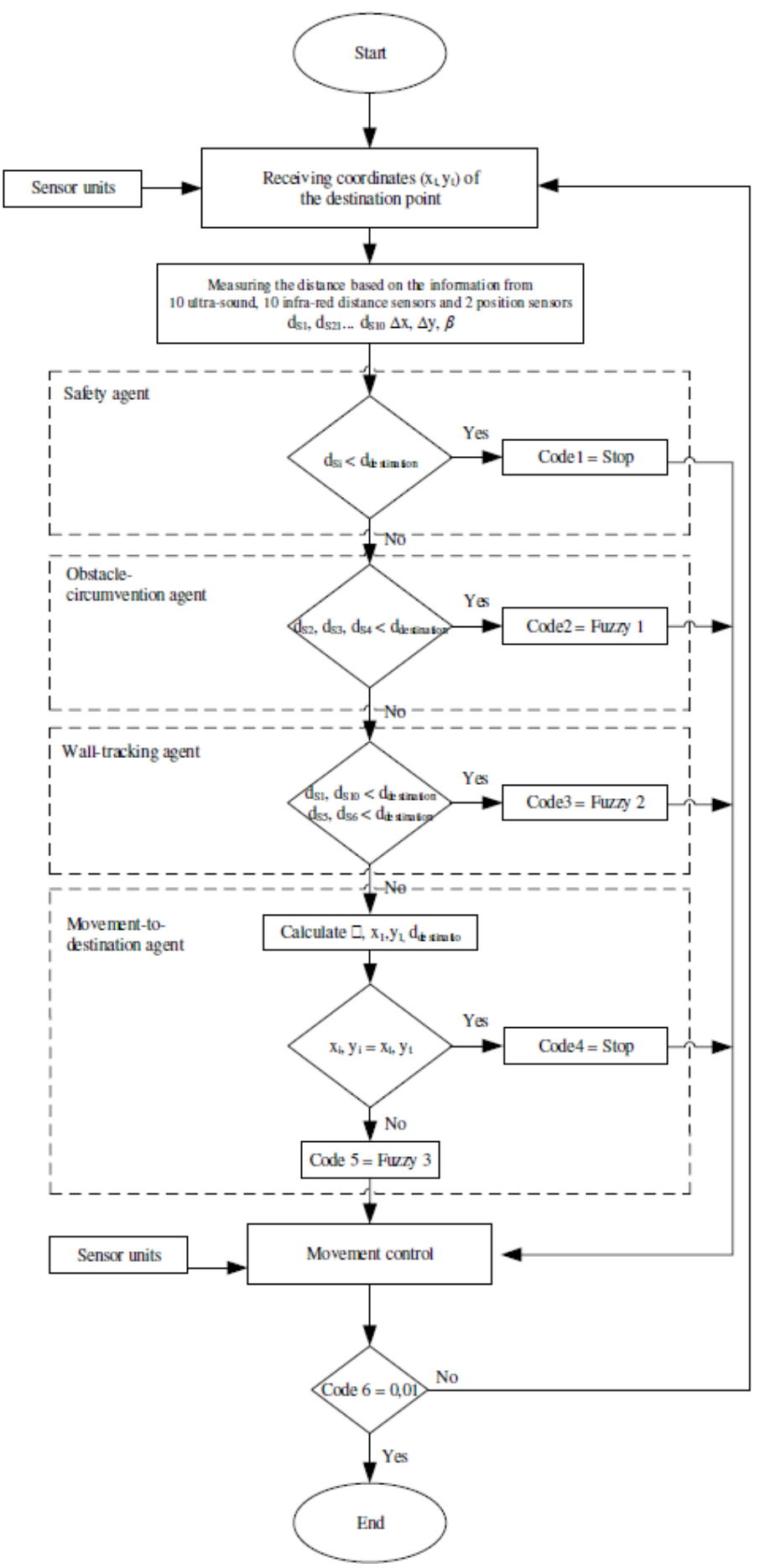

Fig. 2. Operational control algorithm for mobile robot movement in unknown environment 
The intelligent system for planning the mobile robot movement (Fig. 2) is composed of five agents. Beside the four agents, i.e. obstacle-bypass, wall-tracking, movement-to-destination and speed control, one more - safety agent - is used to ensure that the mobile robot does not collide into obstacles while moving. Safety agent gives the STOP command and stops the robot, when an obstacle occurs on the robot's movement trajectory in the so-called "safety zone" (dsafe $=0.10 \mathrm{~m})$. Safety agent has the highest degree of priority.

\section{Results and discussion}

Experimental research on an intelligent system for planning and managing mobile robot movement in an unknown environment. Experimental research on an intelligent system for planning the mobile robot movement in unknown environment was performed in a room with a size of $3 \times 2 \mathrm{~m}$. Position of the initial and destination points in Fig. 3. Default coordinates of the centre of the robot located in the middle of the axis of the two rear wheels are A $(0,0)$. The first destination point $\mathrm{B}(-1$; $0.5)$, second destination point $\mathrm{C}(-0.5 ; 1.5)$ and the third cycle point $\mathrm{D}(1 ; 1)$. Control programs are written in the Arduino software language.

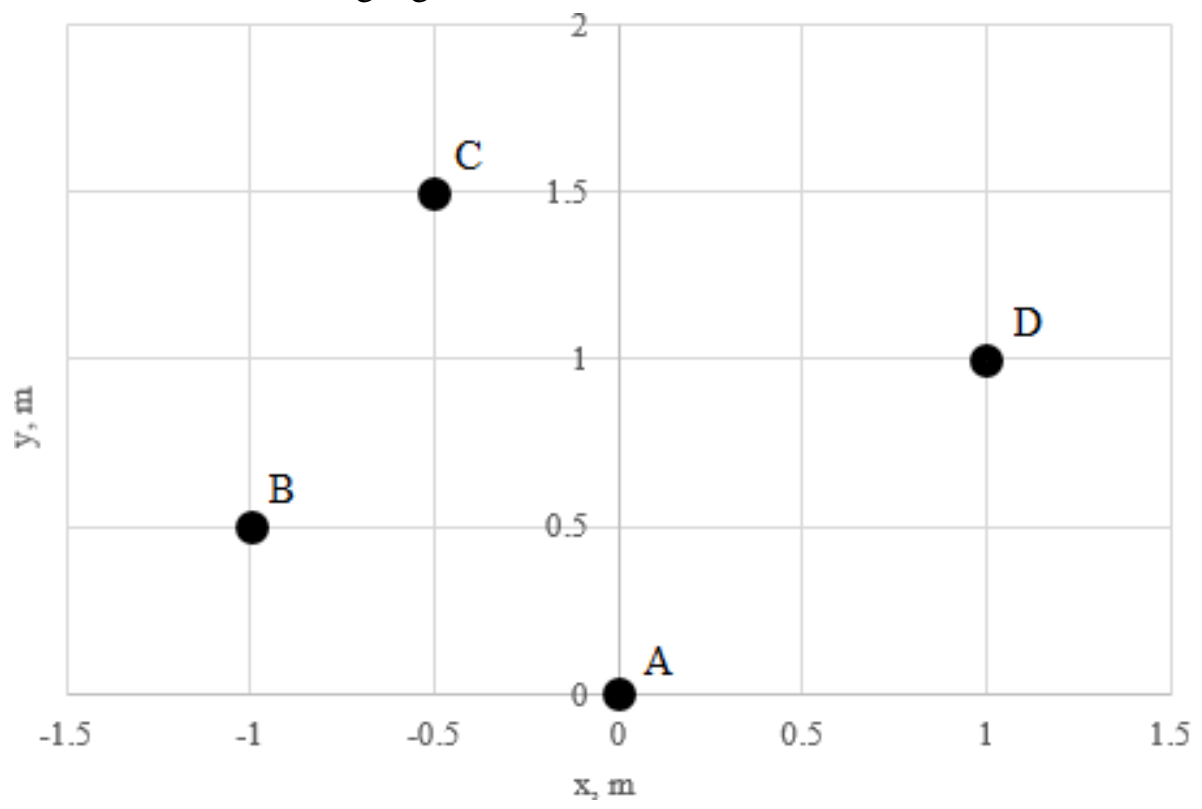

Fig. 3. Position of initial and destination points

\section{Experimental research for the movement-to-destination agent}

The mobile robot was moving from the initial point $\mathrm{A}$ to the destination point $\mathrm{B}(-1 ; 0.5)$. The results of the practical experiment and movement trajectory are presented in Fig. 4. After 1981 iterations of the program (the set time is $58.4 \mathrm{~s}$ ) the robot reached the destination without colliding with an obstacle.

\section{Experimental research for the wall-tracking agent}

The mobile robot was moving from the initial point A to the destination point B $(-1 ; 0.5)$. After 2659 iterations of the program (the set time is $74.8 \mathrm{~s}$ ) the robot reached the destination. The results of the practical experiment and movement trajectory in the movement-to-destination agent are presented in Fig. 5.

Experimental research on the planning system for mobile robot movement in an unknown environment

During the first experiment the mobile robot was moving from the initial point $\mathrm{A}$ to the destination point $\mathrm{C}(-0.5 ; 1.5)$. After 2139 iterations of the program (the set time is $60.8 \mathrm{~s}$ ) the robot reached the destination without colliding with an obstacle. The results of the practical experiment and mobile robot movement trajectory in the movement-to-destination agent are presented in Fig. 6. 
1)

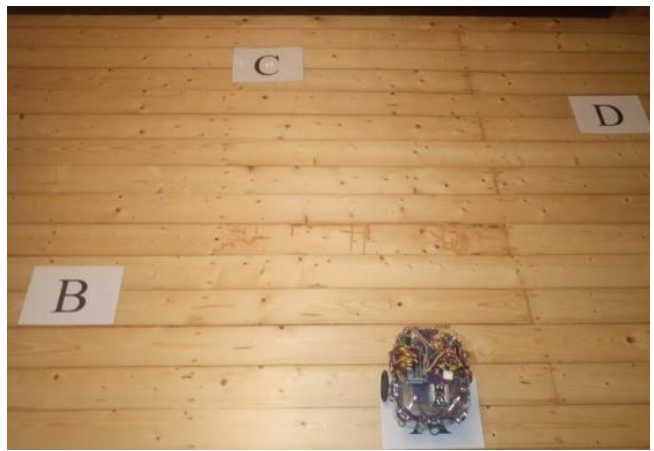

3)

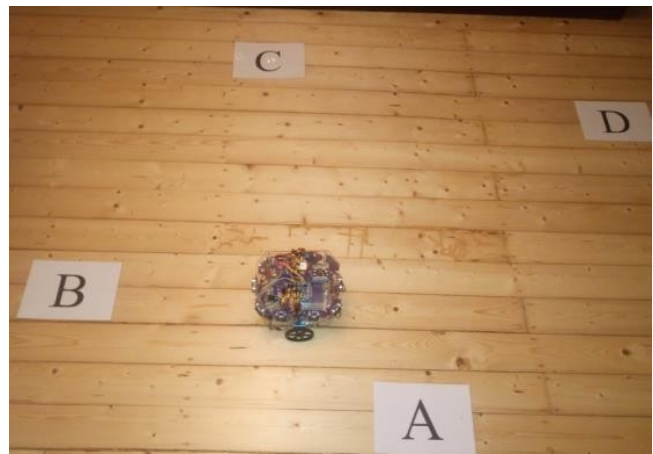

2)

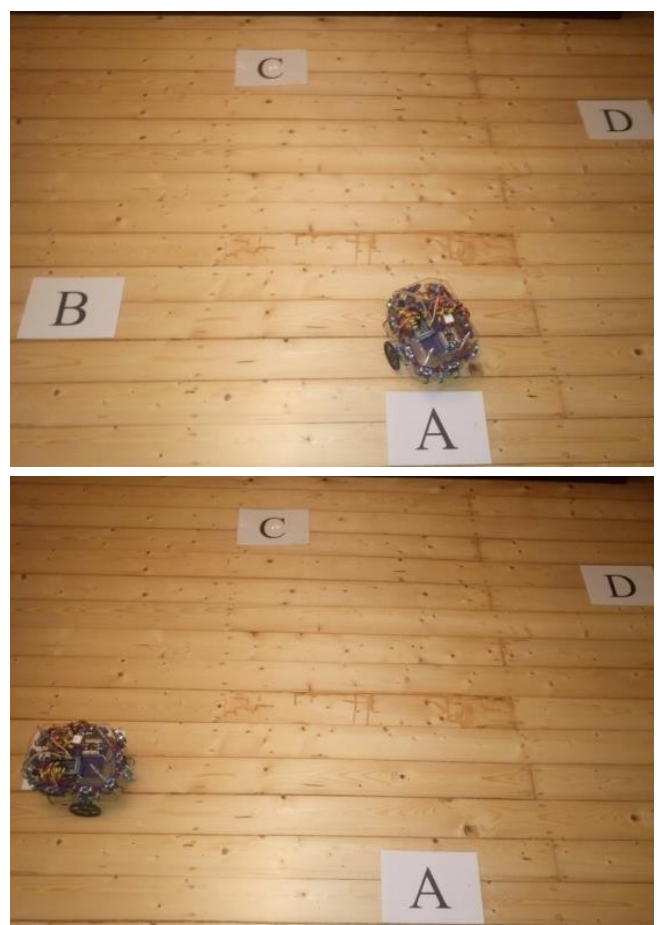

Fig. 4. Results of practical experiment for movement-to-destination agent

1)

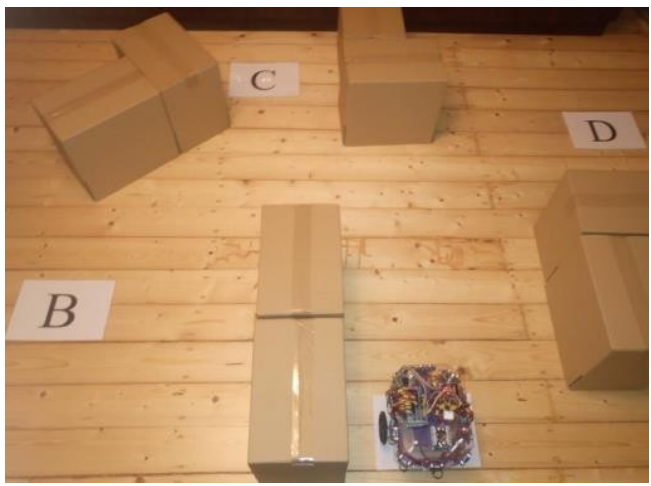

3)

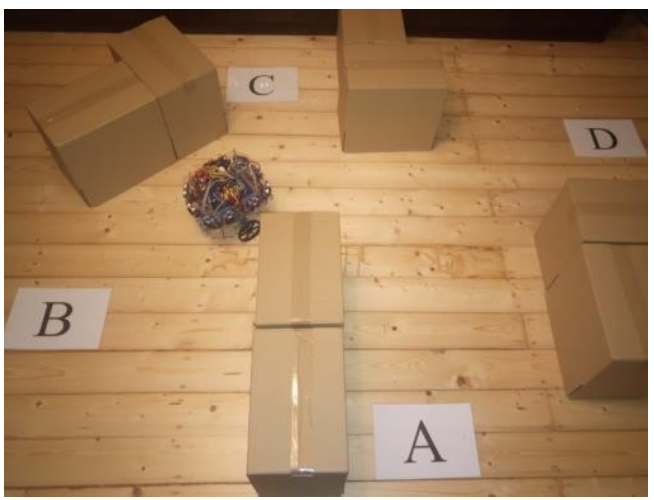

2)

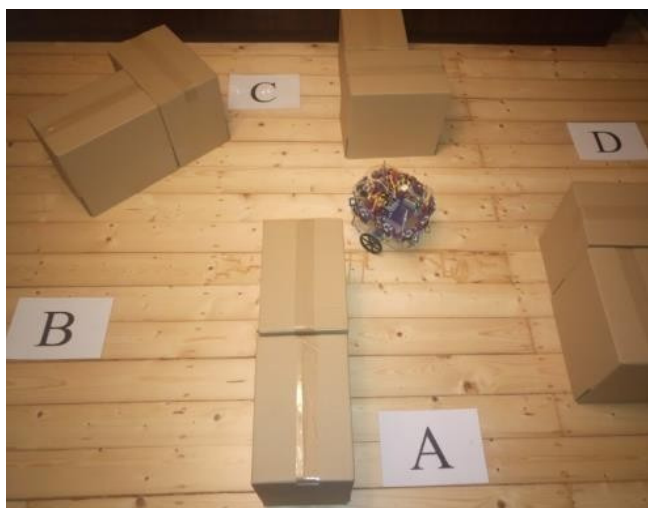

4)

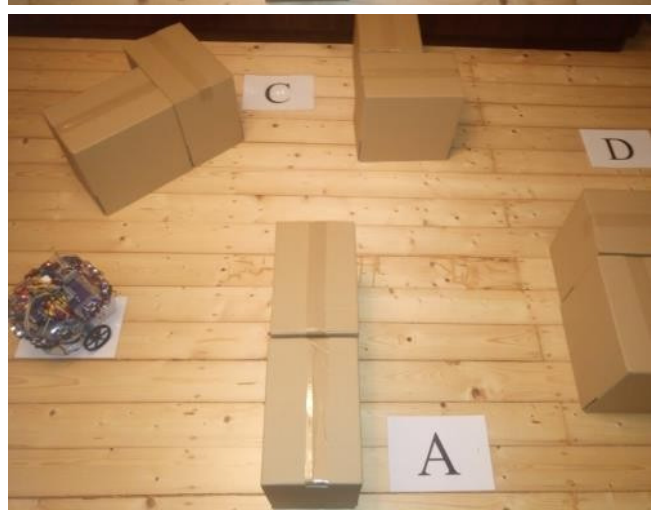

Fig. 5. Results of practical experiment in wall-tracking agent

During the second experiment the mobile robot was moving from the initial point $\mathrm{A}$ to the destination point $\mathrm{D}(1 ; 1)$. After 2496 iterations of the program (the set time is $75.3 \mathrm{~s}$ ) the robot reached the destination. The results of this experiment and mobile robot movement trajectory in the movement-to-destination agent are presented in Fig. 7. 
1)

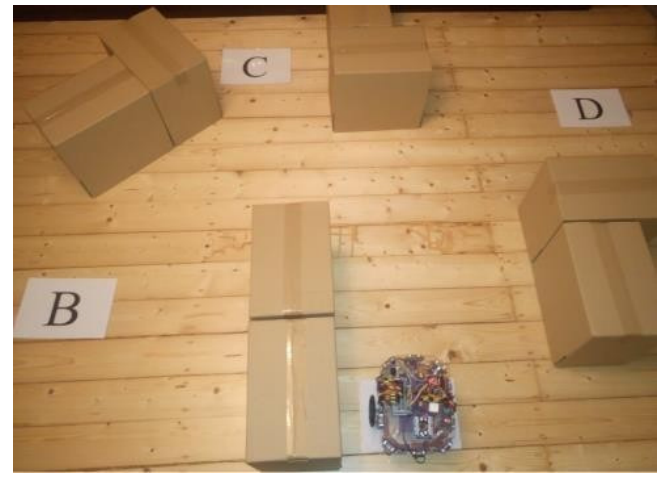

3)

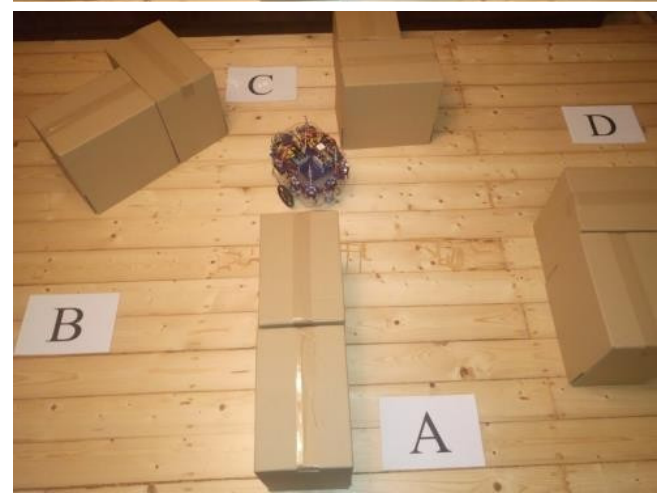

2)

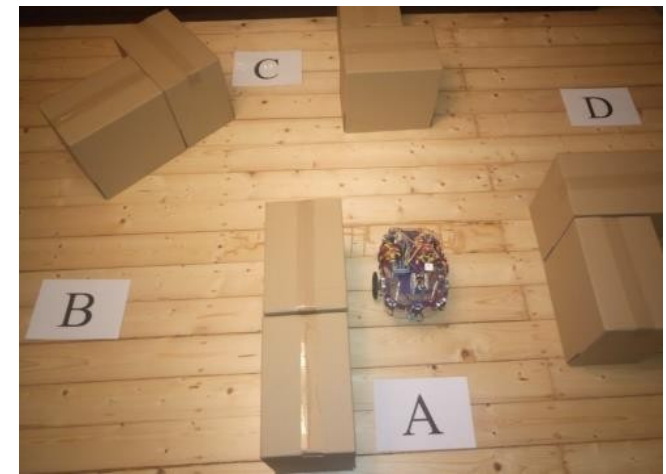

4)

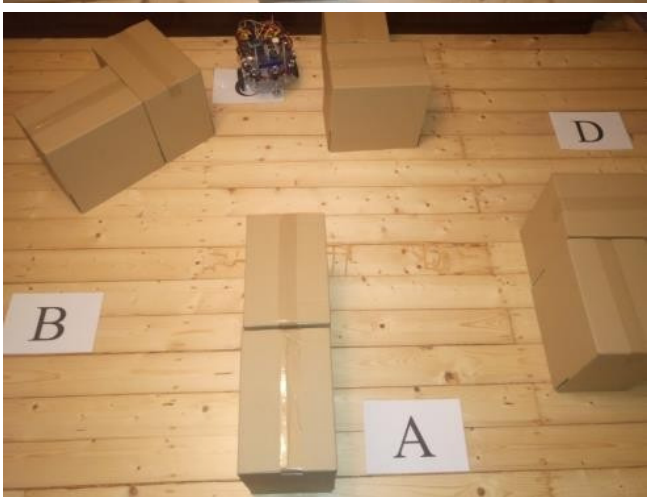

Fig. 6. Results of the first practical experiment in unknown environment

1)

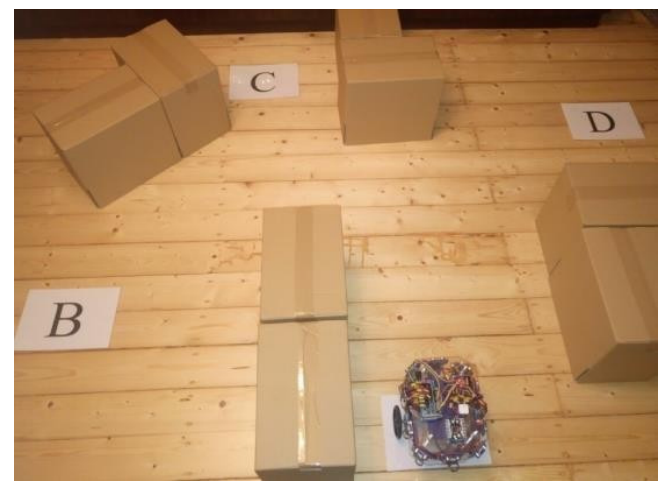

3)

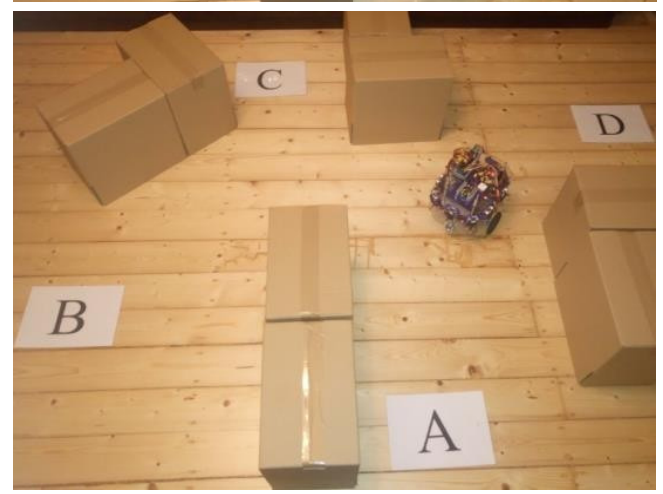

2)

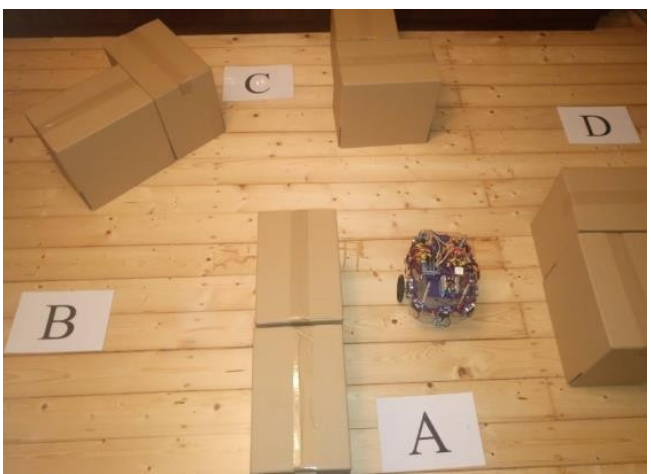

4)

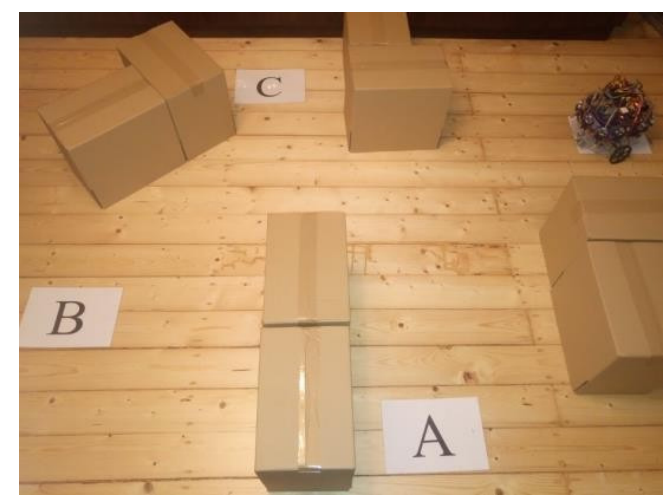

Fig. 7. Results of the second practical experiment in unknown environment

\section{Conclusions}

Following the experimental study of the mechatronic planning system of a mobile robot movement in an unknown environment, the following conclusions can bedrawn:

1. Having practically tested the theoretical operational control systems for mobile robot movement in an unknown environment, their operational effectiveness was confirmed. For the research, an experimental scheme was designed consisting of a four-wheeled mobile robot laboratory model 
with the installed ten infra-red distance sensors and ten ultra-sound distance sensors. The input and output board with micro-controller transmit data to a computer through a serial port (USB cable) or a wireless connection module. Operational control algorithm designed in the Arduino software environment operating in the Windows operating system. Outputs of this algorithm are the mobile robot platform position change commands transmitted directly through the input and output board to the driving motors enabling the robot to move along a "safe" trajectory.

2. The practical experiments performed under conditions of unknown environment with certain obstacles set out on the mobile robot's route in random places unknown to the robot showed that the robot successfully avoidedcollisions.

3. To improve the experimental research base, the following is required: first, larger number of distance sensors with faster and more accurate distance measurements, as this would reduce the respective time costs; second, the structure of the input and output board needs faster microcontroller and more advanced programming language; and third, electronic compass for correcting direction errors. This would also increase the speed and accuracy of the mechatronic system.

\section{References}

[1] Koubaa A., Bennaceur H., Chaari I., Trigui S., Ammar A., Sriti M.F., Alajlan M., Cheikhrouhou O., Javed Y. Robot Path Planning and Cooperation. Foundations, Algorithms and Experimentations. Springer, 2018. 190 p.

[2] Luo C. Neural-network-based fuzzy logic tracking control of mobile robots. Proceedings of International conference "Automation Science and Engineering (CASE)", August 20-23, 2017 Xi'an, China, pp. 1318-1319.

[3] Tian W. The research into methods of map building and path planning on mobile robots. Proceedings of International conference "2017 IEEE 2nd Information Technology, Networking, Electronic and Automation Control Conference (ITNEC)", December 15-17, 2017, Chengdu, China, pp. 1087-1090.

[4] Cardenas A.M., Rázuri J.G., Sundgren D., Rahmani R. Autonomous Motion of Mobile Robot Using Fuzzy-Neural Networks. Proceedings of International conference "Artificial Intelligence", November 24-30, 2013, Mexico City, Mexico, pp. 80-84.

[5] Li Z., Deng J., Lu R., Xu Y., Bai J., Su C.Y. Trajectory-tracking control of mobile robot systems incorporating neural-dynamic optimized model predictive approach. IEEE Transactions on Systems, Man, and Cybernetics: Systems, vol. 46, no. 6, 2016, pp. 740-749.

[6] Dewi T., Risma P., Oktarina Y., Roseno M.T. Neural network controller design for a mobile robot navigation; A case study. Proceedings of International conference "Electrical Engineering, Computer Science and Informatics (EECSI)", September 19-21, 2017, Yogyakarta, Indonesia, pp. $1-5$.

[7] Boujelben M., Ayedi D., Rekik C. and Derbel N. Fuzzy logic controller for mobile robot navigation to avoid dynamic and static obstacles. Proceedings of International conference "Systems, Signals \& Devices (SSD)", March 28-31, 2017, Marrakech, Morocco, pp. 293-298.

[8] Wang H., Duan J., Wang M., Zhao J., Dong Z. Research on Robot Path Planning Based on Fuzzy Neural Network Algorithm. Proceedings of International conference "Information Technology, Electronic and Automation Control Conference (IAEAC)", May 28-30, 2017, Chongqing, China, pp. 1800-1803.

[9] Pandey A., Sonkar R.K., Pandey K.K., Parhi D.R. Path planning navigation of mobile robot with obstacles avoidance using fuzzy logic controller. Proceedings of International conference "Intelligent Systems and Control (ISCO)", January 10-11, 2014, Coimbatore, India, pp. 39-41.

[10]Zhao T., Wang Y. A Neural Network Based Mobile Robot Autonomous Tracking System. Proceedings of International conference "SoutheastCon 2018", April 19-22, 2018, St. Petersburg, FL, USA, pp. 1-2. 